\title{
Pathological

\section{Comparison of multiplex ligation-dependent probe amplification and qPCR for screening PAX5 gene detection in acute lymphoblastic leukemia}

\author{
Sahar Mehranfar ${ }^{1,2}$, Sirous Zeniali ${ }^{3,4}$, Nasser Samadi ${ }^{5}$ Nazanin Maleki Sadeghi ${ }^{6}$ and \\ Abbasali Hosein Pour Feizi ${ }^{* *}$ \\ ${ }^{1,7}$ Hematology \&t Oncology Research Center, Tabriz University of Medical Sciences, Tabriz, Iran \\ ${ }^{2}$ Department of Molecular Medicine, School of Advanced Medical Science, Tabriz University of Medical \\ Science, Tabriz, Iran \\ ${ }^{3}$ Department of Molecular Medicine, Biotechnology Research Center, Pasteur Institute of Iran, Tehran, Iran \\ ${ }^{4}$ Kawsar Human Genetics Research Center, Kawsar Genomics Center, Tehran, Iran \\ ${ }^{5}$ Department of Biochemistry, Faculty of Medicine, Tabriz University of Medical Sciences, Tabriz, Iran \\ ${ }^{6}$ Lorestan University of Medical Sciences, Khorramabad, Iran
}

\begin{abstract}
Despite the advances in diagnosis of leukemia, still we need a rapid and cost-benefit screening method in patients with acute lymphoblastic leukemia (ALL). The aim of the present study is to evaluate the ability of Multiplex Ligationdependent Probe Amplification (MLPA) method in screening patients with ALL. 45 patients with ALL were selected. DNA were extracted, then PAX5 gene copy number abnormalities (CNAs) were studied by adopting MLPA and QPCR methods. To prove the results of two methods, PCR product from three samples were sent for sequencing. From 45 patients with B-ALL, 11 (24\%) patients, showed CNAs after applying MLPA method. From among all mutations, 22 samples (29\%) were seen in PAX5 gene. We used Sanger sequencing as a gold standard method to compare the two methods. After sequencing, we were submitted 2 genes in Gene Bank by accession numbers (ID) KX608846 and KX707789 in exon 10 of PAX5. MLPA is a rapid and valid method for screening of genes mutation and can be used in these laboratories as routine method especially in low-income countries.
\end{abstract}

KEY WORDS: ACUTE LYMPHOBLASTIC LEUKEMIA, COPY NUMBER ABNORMALITY, MLPA, QPCR

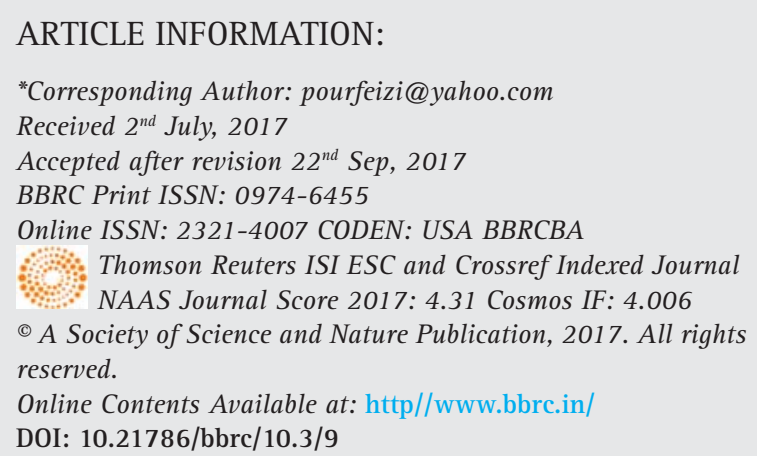




\section{INTRODUCTION}

Today, B-cell acute lymphoblastic leukemia (B-ALL) is the most common childhood malignancy, especially in children below five years of age. Today, we have extensive knowledge about leukemia like other cancers._In the last two decades, molecular methods have provided the physicians and researchers with a new vision towards the role of molecular factors in pathogenesis of diseases. Alternative expression leads to the abnormal proliferation and differentiation of lymphoid ancestors (Fazio, Biondi, \& Cazzaniga, 2011; O'neil \&t Look, 2007 Seiter et al., 2014).

Paired Box5 (PAX5) is an important transcription factor located on chromosome $9 \mathrm{p} 13$ and includes 10 exons. PAX5 is essential factor for the development of pro-B cell to mature B-cell(Fazio et al., 2011). Up to now, nine PAX transcription factors have been demonstrated, although, PAX5 is the only PAX protein which is expressed in the hematopoietic system (Busslinger, 2004; Cobaleda, Schebesta, Delogu, \&t Busslinger, 2007; Matthias \&t Rolink, 2005). The improvement of diagnostic and treatment protocols by using risk-adapted methods have enhanced cure rate up to 80\%(Carter et al., 2001).

Therefore, the identification of biomarkers can be a great help for the patients and the health-care system to reduce the health-care costs and side effects of chemotherapy. Screening is highly pivotal in the developing countries where the financial resources and drugs are limited. Childhood cancer rates has been rising slightly in the past few decades (Fathi et al., 2015).

In Iran, leukemia has an increasing trend and leaves a heavy burden on the whole society,(Fazeli et al., 2013). Thus, more attention needs to be paid to screening, early diagnosis, and effective treatment in order to increase the survival rate for children's cancer (Mousavi et al., 2008).

In this study, we carried out Multiplex Ligation-dependent Probe Amplification (MLPA) analysis on 45 ALL patients' samples to determine the copy number abnormality (CNA) of PAX5 gene. MLPA is a technique capable of showing variations in the copy number of several human genes.Due to this capability, MLPA is mostly used in the molecular diagnosis of several genetic diseases whose pathogenesis is related to the presence of deletions or duplications of specific genes,(Schouten et al., 2002; Stuppia, Antonucci, Palka, \&t Gatta, 2012). Also, we adopted the real-time PCR method for comparing the results of two methods.

\section{MATERIALS AND METHODS}

58 Bone marrow(BM) or/and peripheral blood (PB) samples were taken at the time of diagnosis of patients with B-ALL, who were presented to the Children's Hospital of Tabriz University of Medical Sciences, between 2014 and 2016. The selection criteria included having at least
$20 \%$ blast cells, and being between 1-14 years of age at the time of diagnosis. The exclusion criteria were the samples from foreign patients and DNA with insufficient quality. Six samples were excluded due to failure of MLPA, Five samples due to bad quality of DNA and two of them were withdrawn. Additionally, 4 bone marrow and 7 peripheral blood samples from healthy donors were analyzed as control samples. Graphical abstract of diagnosis process in this study are shown in (Figure 1).

The written informed consent was obtained from all the parents and the study protocol was approved by Ethics and Human Rights Committee of Tabriz University of Medical Sciences.

The DNA was extracted from BM or PB samples, using the QIAamp DNA minikit (Qiagen, Hilden, Germany) according to the manufacturer's instructions. In order to verify the fidelity of DNA concentration and to evaluate its quality, a spectrophotometer,(NanoDrop,Wi lmingtonDE,,USA) and 1.5\% agarose electrophoresis gel were used respectively.

Two pairs of primer for exone 10 of PAX 5 gene were designed using the online program oligo7. The primer sequences are shown in (Table 1). SYBR Green Real-time PCR (BI0-RAD iQ5, Bio-Rad, Hercules, California, USA) was done using a serial dilution of DNA samples including 100 , 50, 25, 12.5 and $6.25 \mathrm{ng}$ in triplicate repeats. Then, according to the standard curves and by comparing the slope and efficiency of each reaction (Schmittgen, Lee, \&t Jiang, 2008), 50 ng of DNA were preferable as the best concentration. The analysis was performed in a total volume of $20 \mu \mathrm{l}$ including $50 \mathrm{ng}$ of DNA, 0.2 $\mathrm{mM}$ specific primers and $10 \mu \mathrm{l}$ of SYBR Green Master mix (Applied Biosystems, Foster City, California, United States) according to the manufacturer's instructions, then $\left(2^{-\Delta \Delta C t}\right)$ was calculated by normalizing samples and the relative gene copy numbers were calculated. The

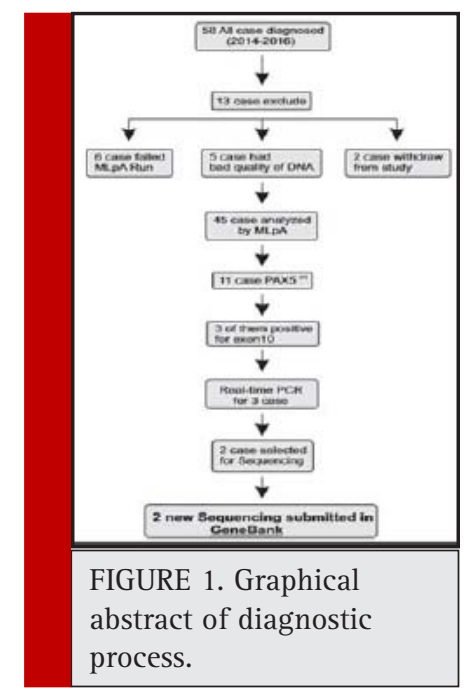




\begin{tabular}{|l|c|c|l|}
\multicolumn{5}{|l|}{ Table 1. The primer sequencing used in QPCR for PAX5 copy number abnormalities. } \\
\hline Gene name & Product length & $\mathrm{Tm}\left(\mathrm{C}^{\circ}\right)$ & Primer sequence \\
\hline PAX5 F1 & 170 & 64 & 5-ATCTTAACCTAGGCAGAGCATC-3 \\
\hline PAX5 R1 & 170 & 61 & 5-CAAGAGACACACCATTTGGG-3 \\
\hline PAX5 F2 & 164 & 62 & 5-CTCCTTCTTAGTATCTACGAG-3 \\
\hline PAX5 R2 & 164 & 60 & 5-GAACTCAAAGAAACTGTCTGG-3 \\
\hline GAPDH F & 120 & 60 & 5-CGGTGGATCCCTTTAATTG-3-3 \\
\hline GAPDH R & 120 & 58 & 5-CAATAAAGGGGATCCACCG-3 \\
\hline
\end{tabular}

copy numbers compared to the reference gene were determined. Following values were considered for result analysis: 1 for normal samples, 0.5 for heterozygous deletions, and 1.5 for heterozygous duplications. Results were analyzed using Microsoft Excel.

The MLPA probe sizes, chromosomal position and sequences and other complete details are available on the Website (www.MRC-Holland.com). The sensitivity of the MLPA kit P335-B2 was assessed previously by Kuiper et al (Kuiper et al., 2007) . In each run of MLPA, appropriate numbers of the samples, and three to six control samples were used in same run. All control samples were taken from the children with no acute leukemia or other types of malignancy. In this study, $50 \mathrm{ng}$ of genomic DNA in final volume of $5 \mu$ l were used to determine copy number of PAX5 gene by using the SALSA MLPA kit P335-B2 (MRC Holland, The Netherlands). Capillary electrophoresis were run on 3130XL Genetic Analyzer (Life Technologies, Carlsbad, CA, USA) and amplified PCR products were analyzed by Gene Marker version 1.95 (Soft Genetics State College, Pennsylvania, USA). Values over $<0.75$ and $>1.35$ were considered to be abnormal and values below 0.25 indicated biallelic deletion. Schematic MLPA analysis is shown in (Figure 2).

Normality of data was calculated by KolmogorovSmirnov test and was presented by Mean and Standard Deviation. Non-normal variables were incidental as Median (Max \& Min). The gene CNAs difference between the ALL and control samples were calculated by independent sample T-test and regression tree for accuracy of MLPA method. P-values less than 0.05 were considered statistically significant. All statistical analyses were done using the Statistical package for the Social Sciences (SPSS), version 16.0 (SPSS Inc, Chicago, IL, USA).

\section{RESULTS AND DISCUSSION}

45 patients with ALL, including 27 (60\%) boys with mean age of $5.8 \pm 3.4$ and $18(40 \%)$ girl with mean age of $6.0 \pm 2.7$ years were studied ( $>>0.05$ ). Statistically, no significant differences were seen between the mean ages of genders. Demographic data of the participants have been summarized in (Table 2).

We found a wide range of alterations in the PAX5 gene in this study; deletion or duplication in one or more exons. In 2 cases, deletion and duplication were seen in different exons simultaneously. From among all patients, 11 (24\%) patients showed 76 CNAs, from which, 22 (29\%) were seen in PAX5 gene. The more frequent exons were 6,7 , and 8 (55\%); the least one was exon 1 (4\%). No significant difference was observed between CNAs and sex, age, National Cancer Institute (NCI) risk group or source of samples $(\mathrm{p}<0.05)$. Schematic detected CNAs are shown in (Figure 3).

From among all samples, three samples showed changes in exon 10 by MLPA. All of these samples (3) were validated by real-time PCR. The standard melting curve of real-time PCR is shown in (Figure 1).

The accuracy obtained for MLPA method for detection of CNAs comparisons of real-time PCR was $86 \%$.

To prove the results of two methods, PCR product from these patients was sent to Bioneer for sequencing. The sequencing analyses were done by Chromas software, and the results were compared with the reported gene sequences. We submitted 2 genes in GenBank by accession numbers (ID) KX608846 and KX707789 in exon 10 of PAX5. Schematic representation of each method is shown in (Figure 4).

ALL is a heterogeneous disease with differing in response to chemotherapy. The results of some studies demonstrated that the identification of molecular markers may improve the treatment approaches (Yang et al., 2011). Today, the determination of gene dosage is important for both clinical and research medicine and it is also required for therapeutic, prognostic, or diagnostic goals (Ginsburg \&t Willard, 2009; Vogelstein \&t Kinzler, 2004). Due to the growing need for molecular studies, a lot of new molecular markers have been discovered (Mrózek, 


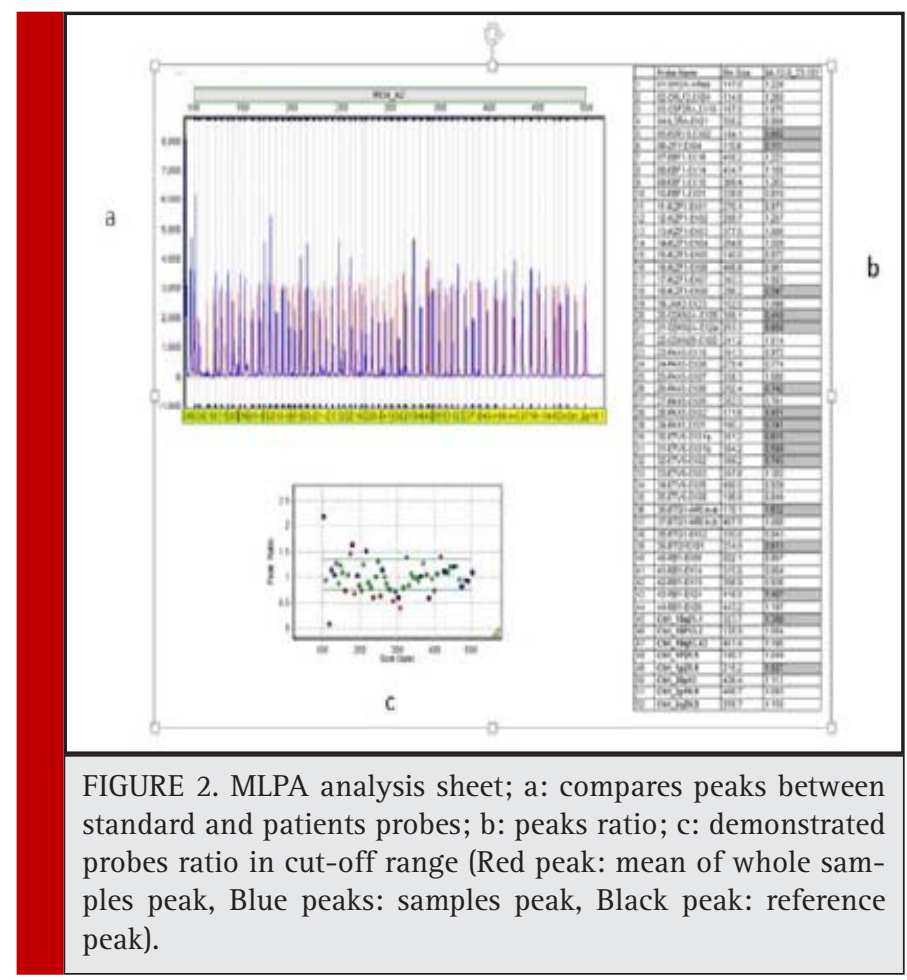

Döhner, Et Bloomfield, 2007; Thiede et al., 2006). It seems that in the future, patients could be divided into two groups in terms of presence or absence of these biomarkers (Mi et al., 2012).

PAX5 translocations do not influence the prognosis or outcome by themselves but it is in association with other molecular aberrancies in theirs pathway that they influence the prognosis or outcome (Sellner $\mathrm{Ct}$ Taylor, 2004). Similar to our findings, the incidence of PAX 5 rearrangements was seen about 30\% in the B-cell precursor ALL, in a previous study (Barbosa et al., 2015; Hunger Ct Mullighan, 2015; C. Schwab et al., 2010; C. J. Schwab et al., 2013). Array-based approaches can provide high resolution data on CNAs, but these methods are restricted by low throughput, high cost, and time consumption. Therefore, the need for a simple, fast and cost-efficient method to screen molecular changes has remained hitherto. (Vermeesch et al., 2007).

The PCR-based multiplex ligation-dependent probe amplification (MLPA) technique is a relative quantification method for gene dosage determination and deletions/duplications mutations in unknown samples which are recognized by comparison to the normal and standard control (Al Zaabi, Fernandez, Sadek, Riddell, Et Greer, 2010). Buijs et al. performed genomic profiling using MLPA in 45 cases with (ALL), showing that MLPA is able to detect anomalies similar to PCR method (Buijs, Krijtenburg, \& Meijer, 2006).

A similar study was done by Coll-Mulet et al., who carried out MLPA in 50 chronic leukemia patients. Their results illustrated that only cases with a low percentage $(<25 \%)$ of cells, were not detected by MLPA (Coll-Mulet et al., 2008). Many other studies on different types of leukemia revealed excellent accuracy and specificity of MLPA as compared to QPCR and showed clinical capability of these techniques with different disease(Abdool, Donahue, Wohlgemuth, \&t Yeh, 2010; Donahue, Abdool, Gaur, Wohlgemuth, \&t Yeh, 2011; Fabris et al., 2011; Mullighan et al., 2008; C. Schwab et al., 2010; Schwartz \& Dunø, 2004).

However, there are still some limitations such as being unable to detect balanced rearrangements, mosaicism, heterogeneity or contamination with normal cells. Like PCR, MLPA reactions are also more sensitive to contaminants (Stuppia et al., 2012; Tavtigian \& Le Calvez-Kelm, 2007). According to the manufacturer's instructions of MLPA, it works with only $20 \mathrm{ng}$ of DNA. Besides, the results don't depend on the amount of used sample DNA but, our study showed that $50 \mathrm{ng}$ are required for reliable results.

Moreover, it is recommended to compare different MLPA analyses only by using one DNA extraction method from different sources of samples from each patient, to compare specify the sensitivity of this method for different sources of samples. Real-time PCR method is highly sensitive and specific as reported in many studies all over the world, though, it is a time-consuming method and can survey only one target sequence in per run (Ponchel et al., 2003; Ramalingam et al., 2009).

But Compared to QPCR, MLPA is a low cost, fast, and technically uncomplicated method for the analysis of 


\begin{tabular}{|l|l|c|}
\hline \multicolumn{3}{|l}{ Table 2. Demographic data of acute lymphoblastic } \\
leukemia patients & Frequency (Percent) \\
\hline \multirow{2}{*}{ Variable } & Female & $18(40 \%)$ \\
\cline { 2 - 3 } & Male & $27(60 \%)$ \\
\hline \multirow{2}{*}{ Age } & $1-4$ & $22(48 \%)$ \\
\cline { 2 - 3 } & $5-10$ & $17(37 \%)$ \\
\cline { 2 - 3 } & $10-14$ & $6(13 \%)$ \\
\hline \multirow{2}{*}{ Mean of age } & Female & $6.0 \pm \pm 2.7$ \\
\cline { 2 - 3 } & Male & $5.8 \pm 3.4$ \\
\hline \multirow{2}{*}{ NCI risk group } & Standard risk & $28(62 \%)$ \\
\cline { 2 - 3 } & High risk & $17(37 \%)$ \\
\hline \multirow{2}{*}{$\begin{array}{l}\text { Source of } \\
\text { samples }\end{array}$} & BM samples & $19(42 \%)$ \\
\cline { 2 - 4 } & PB samples & $26(57 \%)$ \\
\hline \multirow{2}{*}{ WBC count } & $>50,000$ & 11 \\
\cline { 2 - 4 } & $<50,000$ & 34 \\
\hline
\end{tabular}

results. Moreover, in the future, MLPA could be applied to large CNAs screening. PCR and sequencing can be used for confirming mutation only in selected gene segments, but MLPA can be considered for screening a large area of genes, simultaneously.

\section{CONCLUSION}

Molecular screening is an essential test for comprehensive survey of disease, and provides complementary information for better diagnosis, treatment and followup of patients with acute leukemia. This approach may be useful as a criterion for measuring the effectiveness of new molecular tools such as genetic profiling. Considering differences between two methods, more clinical studies are required to find out the best one for our objectives. Our data clearly indicated that MLPA can be an attractive alternative method to other molecular and cytogenetic techniques that are now routinely used. Screening of critical genes in pathobiology of all cancers such as acute lymphoblastic leukemia could help early diagnosis in the early stages. It is especially useful when the inexpensive and available techniques are used everywhere. It has been established that early diagnosis has a positive impact on the prognosis.

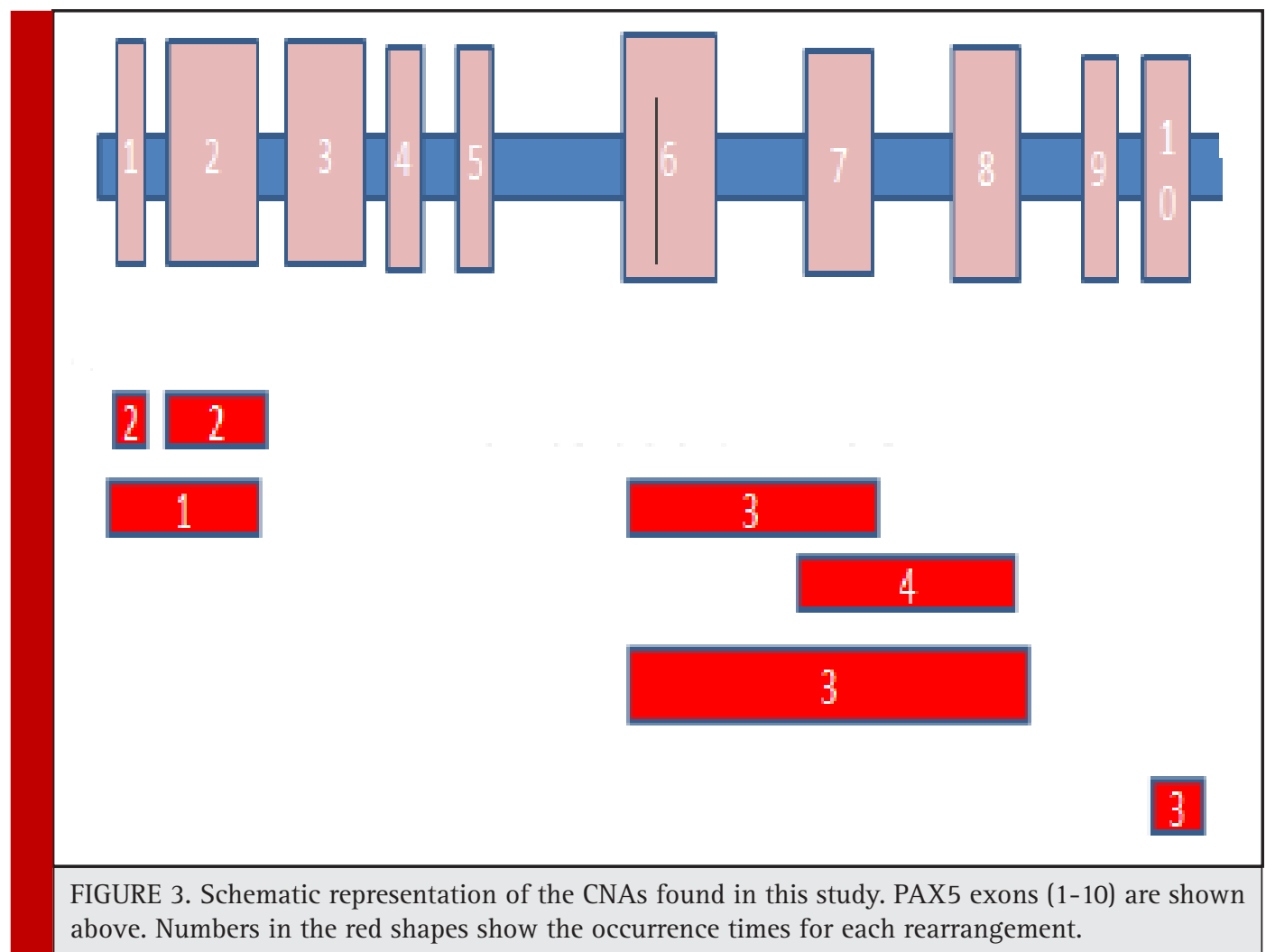




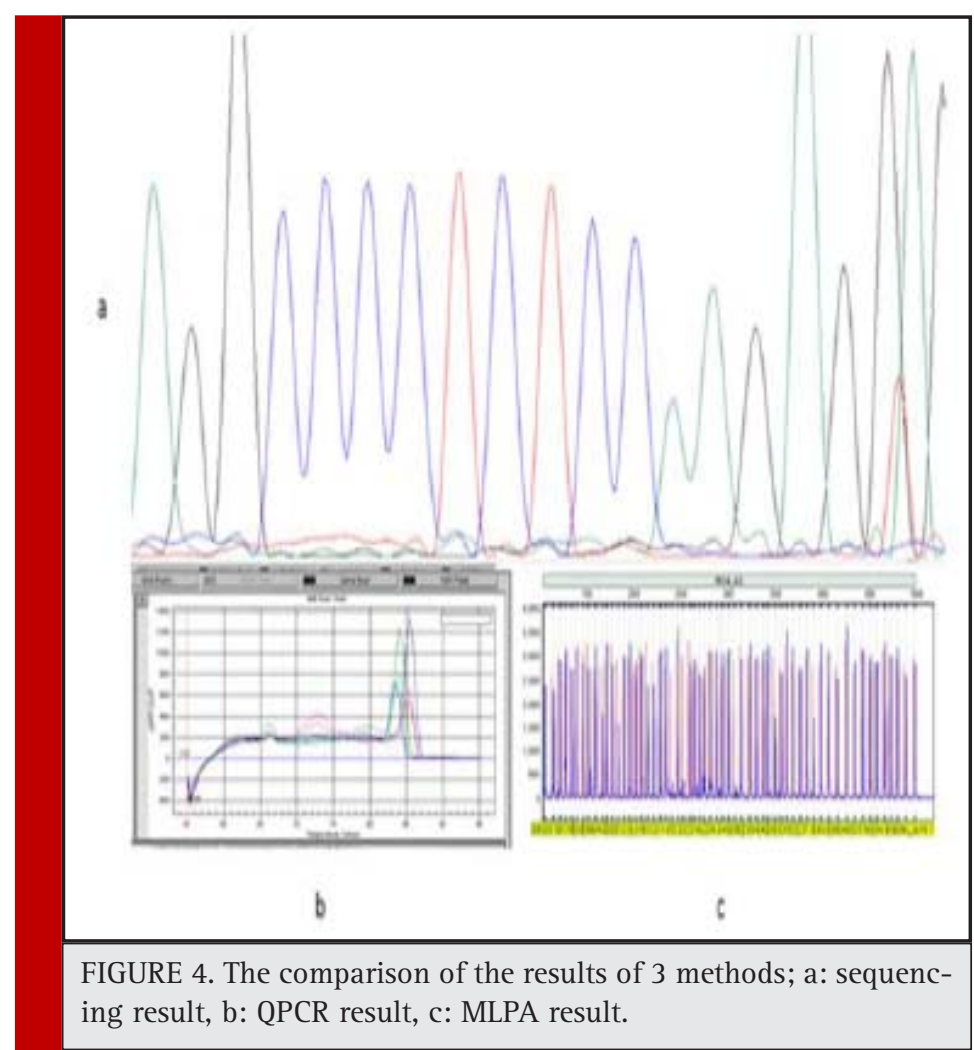

\section{REFERENCES}

Abdool, A., Donahue, A. C., Wohlgemuth, J. G., \& Yeh, C.-H. (2010). Detection, analysis and clinical validation of chromosomal aberrations by multiplex ligation-dependent probe amplification in chronic leukemia. PLoS One, 5(10), e15407.

Al Zaabi, E. A., Fernandez, L. A., Sadek, I. A., Riddell, D. C., \&t Greer, W. L. (2010). Multiplex ligation-dependent probe amplification versus multiprobe fluorescence in situ hybridization to detect genomic aberrations in chronic lymphocytic leukemia: a tertiary center experience. The Journal of molecular diagnostics, 12(2), 197-203.

Barbosa, T. C., Terra-Granado, E., Magalhães, I. M. Q., Neves, G. R., Gadelha, A., Guedes Filho, G. E., . . . Pombo-de-Oliveira, M. S. (2015). Frequency of copy number abnormalities in common genes associated with B-cell precursor acute lymphoblastic leukemia cytogenetic subtypes in Brazilian children. Cancer genetics, 208(10), 492-501.

Buijs, A., Krijtenburg, P. J., \& Meijer, E. (2006). Detection of riskidentifying chromosomal abnormalities and genomic profiling by multiplex ligation-dependent probe amplification in chronic lymphocytic leukemia. Haematologica, 91(10), 1434-1435.

Busslinger, M. (2004). Transcriptional control of early B cell development 1. Annu. Rev. Immunol., 22, 55-79.

Carter, T. L., Watt, P. M., Kumar, R., Burton, P. R., Reaman, G. H., Sather, H. N., Kees, U. R. (2001). Hemizygous p16 INK4A deletion in pediatric acute lymphoblastic leukemia predicts independent risk of relapse. Blood, 97(2), 572-574.
Cobaleda, C., Schebesta, A., Delogu, A., \&t Busslinger, M. (2007). Pax5: the guardian of B cell identity and function. Nature immunology, 8(5), 463-470.

Coll-Mulet, L., Santidrián, A. F., Cosialls, A. M., Iglesias-Serret, D., De Frias, M., Grau, J., Domingo, A. (2008). Multiplex ligation-dependent probe amplification for detection of genomic alterations in chronic lymphocytic leukaemia. British journal of haematology, 142(5), 793-801.

Donahue, A. C., Abdool, A. K., Gaur, R., Wohlgemuth, J. G., \& Yeh, C.-H. (2011). Multiplex ligation-dependent probe amplification for detection of chromosomal abnormalities in myelodysplastic syndrome and acute myeloid leukemia. Leukemia research, 35(11), 1477-1483.

Fabris, S., Scarciolla, O., Morabito, F., Cifarelli, R. A., Dininno, C., Cutrona, G., ... Ciceri, G. (2011). Multiplex ligation-dependent probe amplification and fluorescence in situ hybridization to detect chromosomal abnormalities in Chronic lymphocytic leukemia: A comparative study. Genes, Chromosomes and Cancer, 50(9), 726-734.

Fathi, A., Amani, F., \&t Bahadoram, M. (2015). Epidemiology of Childhood Cancer in Northwest Iran. Asian Pacific Journal of Cancer Prevention, 16(13), 5459-5462.

Fazeli, Z., Pourhoseingholi, M. A., Vahedi, M., Abadi, A., Bavand-Pour, F. S. F., \&t Baghestani, A. R. (2013). Leukemia cancer mortality trend in Iran, from 1995 to 2004. Iranian journal of cancer prevention, 6(3), 170.

Fazio, G., Biondi, A., \& Cazzaniga, G. (2011). The Role of PAX5 in ALL: Citeseer. 
Ginsburg, G. S., \&t Willard, H. F. (2009). Genomic and personalized medicine: foundations and applications. Translational research, 154(6), 277-287.

Hunger, S. P., \&t Mullighan, C. G. (2015). Acute lymphoblastic leukemia in children. New England Journal of Medicine, 373(16), 1541-1552.

Kuiper, R., Schoenmakers, E., Van Reijmersdal, S., Hehir-Kwa, J., van Kessel, A. G., Van Leeuwen, F., \&t Hoogerbrugge, P. (2007). High-resolution genomic profiling of childhood ALL reveals novel recurrent genetic lesions affecting pathways involved in lymphocyte differentiation and cell cycle progression. Leukemia, 21(6), 1258-1266.

Matthias, P., \&t Rolink, A. G. (2005). Transcriptional networks in developing and mature B cells. Nature Reviews Immunology, 5(6), 497-508.

Mi, J., Wang, X., Yao, Y., Lu, H., Jiang, X., Zhou, J. Tang, J. (2012). Newly diagnosed acute lymphoblastic leukemia in China (II): prognosis related to genetic abnormalities in a series of 1091 cases. Leukemia, 26(7), 1507-1516.

Mousavi, S., Alamolhoda, A., Gouya, M., \&t Lickiss, N. (2008). Implementation of Comprehensive National Cancer Control Program in Iran: an experience in a developing country. Annals of Oncology, 19(2), 398-400.

Mrózek, K., Döhner, H., \& Bloomfield, C. D. (2007). Influence of new molecular prognostic markers in patients with karyotypically normal acute myeloid leukemia: recent advances. Current opinion in hematology, 14(2), 106-114.

Mullighan, C. G., Miller, C. B., Radtke, I., Phillips, L. A., Dalton, J., Ma, J., . . Pui, C.-H. (2008). BCR-ABL1 lymphoblastic leukaemia is characterized by the deletion of Ikaros. Nature, 453(7191), 110-114.

0'neil, J., \& Look, A. (2007). Mechanisms of transcription factor deregulation in lymphoid cell transformation. Oncogene, 26(47), 6838-6849.

Ponchel, F., Toomes, C., Bransfield, K., Leong, F. T., Douglas, S. H., Field, S. L. Mighell, A. J. (2003). Real-time PCR based on SYBR-Green I fluorescence: an alternative to the TaqMan assay for a relative quantification of gene rearrangements, gene amplifications and micro gene deletions. BMC biotechnology, 3(1), 1.

Ramalingam, N., Liu, H.-B., Dai, C.-C., Jiang, Y., Wang, H., Wang, Q. Gong, H.-Q. (2009). Real-time PCR array chip with capillary-driven sample loading and reactor sealing for pointof-care applications. Biomedical microdevices, 11(5), 10071020.

Schmittgen, T. D., Lee, E. J., \&t Jiang, J. (2008). High-throughput real-time PCR. Molecular Beacons: Signalling Nucleic Acid Probes, Methods, and Protocols, 89-98.
Schouten, J. P., McElgunn, C. J., Waaijer, R., Zwijnenburg, D., Diepvens, F., \&t Pals, G. (2002). Relative quantification of 40 nucleic acid sequences by multiplex ligation-dependent probe amplification. Nucleic acids research, 30(12), e57-e57.

Schwab, C., Jones, L., Morrison, H., Ryan, S., Yigittop, H., Schouten, J., \&t Harrison, C. (2010). Evaluation of multiplex ligation-dependent probe amplification as a method for the detection of copy number abnormalities in B-cell precursor acute lymphoblastic leukemia. Genes, Chromosomes and Cancer, 49(12), 1104-1113.

Schwab, C. J., Chilton, L., Morrison, H., Jones, L., Al-Shehhi, H., Erhorn, A., . . Harrison, C. J. (2013). Genes commonly deleted in childhood B-cell precursor acute lymphoblastic leukemia: association with cytogenetics and clinical features. Haematologica, haematol. 2013.085175.

Schwartz, M., \& Dunø, M. (2004). Improved molecular diagnosis of dystrophin gene mutations using the multiplex ligationdependent probe amplification method. Genetic testing, 8(4), 361-367.

Seiter, K., Sarkodee-Adoo, C., Talavera, F., Sacher, R., \& Besa, E. (2014). Acute lymphoblastic leukemia. Sarkodee-Adoo C, Talavera F, Sacher RA and Besa EC: Medscape Reference. WebMD. Accessed, 17.

Sellner, L. N., \&t Taylor, G. R. (2004). MLPA and MAPH: new techniques for detection of gene deletions. Human mutation, 23(5), 413-419.

Stuppia, L., Antonucci, I., Palka, G., \&t Gatta, V. (2012). Use of the MLPA assay in the molecular diagnosis of gene copy number alterations in human genetic diseases. International journal of molecular sciences, 13(3), 3245-3276.

Tavtigian, S. V., \& Le Calvez-Kelm, F. (2007). Molecular Diagnostics: Methods and Limitations. Hereditary Breast Cancer, 179-205.

Thiede, C., Koch, S., Creutzig, E., Steudel, C., Illmer, T., Schaich, M., \&t Ehninger, G. (2006). Prevalence and prognostic impact of NPM1 mutations in 1485 adult patients with acute myeloid leukemia (AML). Blood, 107(10), 4011-4020.

Vermeesch, J. R., Fiegler, H., de Leeuw, N., Szuhai, K., Schoumans, J., Ciccone, R. Van Ravenswaaij, C. (2007). Guidelines for molecular karyotyping in constitutional genetic diagnosis. European Journal of Human Genetics, 15(11), 1105-1114.

Vogelstein, B., \& Kinzler, K. W. (2004). Cancer genes and the pathways they control. Nature medicine, 10(8), 789-799.

Yang, Y. L., Hung, C. C., Chen, J. S., Lin, K. H., Jou, S. T., Hsiao, C. C.Lin, S. R. (2011). IKZF1 deletions predict a poor prognosis in children with B-cell progenitor acute lymphoblastic leukemia: A multicenter analysis in Taiwan. Cancer science, 102(10), 1874-1881. 